mounted on a horizontal revolving ring or annular frame, provided with rollers at suitable intervals apart, which roll on a fixed circular track or rail firmly secured to the wall of the building. The dome is made in two halves, divided in a vertical plane through the centre, and hinged at one extremity of this division at two of their adjacent corners to a pin fixed to the revolving ring or annular frame above mentioned. At the opposite corners of the two halves of the dome are fixed rollers, one at each corner, and two other rollers are supplied $90^{\circ}$ from the first pair, one in each half. These rollers roll on the surface of the revolving ring, or on short races, in such a manner as to support the two halves of the dome on the horizontal revolving ring, and permit of their being opened or closed. The opening is, of course, a wedge-shaped one. The two halves of the dome being mounted on the ring, which is capable of revolving by means of the rollers resting on the circular rail, the wedge-shaped opening in the dome can be brought to face any part of the sky."

It may be mentioned as a further advantage of this arrangement that in the winter season, when the dome may be covered with snow, it does not prevent it being opened, as is so frequently the case with domes with shutters; and, furthermore, it gives facility for any design of dome being used, which a shutter renders impossible.

A New Astronomical Periodical. - We have received a circular from the Manora Observatory in Lussenpiccolo, inform. ing us that a new illustrated astronomical periodical will be published from that observatory, with Herr Leo Brenner as editor. Ten numbers will be issued during the year, and these altogether will contain 400 pages and over Ioo illustrations. The price for the ten numbers is stated to be twelve shillings, post free.

\section{CONFERENCE OF THE INTERNATIONAL} GEODETIC ASSOCIATION.1

THE twelfth general conference of the International Geodetic Association was held at Stuttgart on October 3-12, 1898 . Fifteen countries of the nineteen composing the Association were represented. Of the thirty-seven delegates present fifteen came from Germany, five from France, three from Italy, two from Switzerland, two from Japan, and one each from Belgium, England, the Netherlands, Austria-Hungary, Russia, Sweden, Spain, Mexico, and the United States. Besides the delegates, eighteen invited guests appeared, making a total of fifty-five persons present at the meetings. By far the greater part of the time was naturally taken up in reading the regular reports by the delegates on the geodetic work accomplished in their respective countries since the last general conference.

Of special interest was the contribution by Prof. Helmert on the activity of the Central Bureau of the Association at Potsdam. The following work was described: (I) The systematic deviations of the plumb line in connection with the European arc on the $52^{\circ}$ of north latitude. (2) The international latitude service. (3) Absolute determinations of gravity. The international latitude service will be inaugurated during the summer of 1899 at four stations under the direct control and at the expense of the Association. Observations at two additional stations will be undertaken, for which the Central Bureau will supply only a part of the funds necessary. Although the mathematical conditions are not essentially changed by the introduction of Cincinnati and Tschardjui, there is great advantage from the fact that any systematic errors in the regular stations will be more readily discovered. It is a fortunate coincidence that the observatory at Cincinnati happens to be precisely on the parallel of latitude chosen for this work, and it is, moreover, a matter for congratulation that Russia is ready and willing to aid in the undertaking, to the extent of establishing and superintending the station at Tschardjui.

The latest results from pendulum observations are with reference to changes of the force of gravity with elevation, and the derivation of a new formula for the length (L) of the seconds pendulum at the sea-level. From a discussion of over 500 stations the conclusion is reached that what is usually known as continental attraction amounts practically to nothing, and that in general the change of the force of gravity at any point

1 Abridged from an official report by Mr. E. D. Preston to the Superintendent of the Coast and Geodetic Survey, published in Science. on the earth's surface depends purely and simply on the Newtonian law of the inverse square of the distance. The introduction of a spherical function of the third order in the formula for L, alluded to before, foreshadows the determination of a different curvature for the northern and southern hemispheres of the earth; yet the coefficient appears so small that the inequality cannot as yet be safely predicted. The compression given is $1 / 297$, and the equatorial value of the force of gravity is about $1 / 13,000$ greater than that now accepted. The investigation of the relation between disturbances in the force of gravity and deviations of the plumb line is one of the most interesting problems of the near future.

Three matters of universal interest were brought before the Association, and suitable action was taken thereon. These were: (I) "The determination of the figure of the earth by the measurement of arcs and the determination of the force of gravity." (2) "The remeasurement of the Peruvian arc." (3) "The redetermination of the difference of longitude between Paris and Greenwich." The first two subjects were introduced as resolutions by the United States delegate, and brought out interesting discussions. The first was proposed for the sake of directing the attention of the Association more specifically to the prime object of its existence. No one doubts the utility or necessity of a complete study of the law governing the changes of latitude, but its ultimate bearing is rather one of astronomy than geodesy. The funds of the Association are now being devoted largely to the latitude question, and the time seemed fitting to suggest work more directly in the line of its avowed purpose-the measurement of the earth. The object of the resolution was, therefore, in the nature of a recall to the original conception of its being, and bespoke in the interest of pure geodesy an application of its resources to a realisation of the idea of its founders.

The remeasurement of the Peruvian arc now appears to be assured. It may be worth while just here to recall the essential features of this work. One hundred and sixty years ago, when it was a disputed question whether the polar or equatorial diameter of the earth was the longer, the French Academy decided to make one supreme effort to settle the point. To this end, two arcs were measured : one on the equator (now known as the Peruvian arc, although it is really in Ecuador); and the other in Lapland, as near the pole as possible. These two arcs, confessedly inaccurate in the light of modern geodesy, have been employed continually in the determination of the earth's figure. Situated as they are, near the extremities of the quadrant, their influence is great on the shape deduced, so that one of the pressing needs of the day in the measurement of the earth is a redetermination of their lengths. It is proposed to make a reconnaissance during the summer of 1899 , report the results thereof to the Paris conference of 1900 , and then decide definitely on the plans of final measurement. It is universally conceded that France should be given the first chance to act, not alone because the first measure was made by her, but also because the conference of 1889 relinquished in her interest further consideration of the subject.

The Russians and Swedes, in a quiet sort of way, are measuring an arc between the parallels of latitude $77 \frac{1}{2}^{\circ}$ and $81 \frac{1}{2}^{\circ}$ in Spitsbergen. The triangulation will require two summers and part of one winter, and will cost 100,000 kronen (5500l.), exclusive of cost of vessels furnished by the governments. The field work will be finished in I900, and the computations two years later. Fifty stations will be occupied. The sides range in length from ten to one hundred and thirty kilometres, and the bases, of which there will be only two, are to be measured with Jaederin's steel tape line, twenty metres long.

The difference of longitude between Paris and Greenwich came up again for investigation. Nothing seems to remain but to study the conditions of the work of 1888 and 1892, and reconcile, if possible, the outstanding difference. The matter has been referred by the International Geodetic Association to the directors of the two observatories, and a definite result will, doubtless, soon be made known.

The variation of the latitude seems to be at present an absorbing question before the Association; and there results from action taken at the Stuttgart meeting the following status:Six stations will be established during the summer of 1899 on the parallel of latitude $39^{\circ} 8^{\prime}$. These will be distributed in longitude as follows: one each in Japan, Turkestan and Italy, and three in the United States. The American ones will be located at Gaithersburg, Md. ; Cincinnati, O.; and Ukiah,

NO. I 524, VOL. 59] 
Cal. This gives a preponderance of weight to the determination of the coordinate $\mathrm{Y}$ (X passing through Greenwich), but a station in Portugal, which may possibly be secured later, would essentially increase the accuracy of X. Tschardjui, in Russia, and Ukiah, in California, are nearly opposite, and Mizusawa, in Japan, is in the only remaining unoccupied quadrant. The scheme proposed is, therefore, a favourable one for the study of the motion of the pole. No one knows as yet how long it will be desirable to continue the observations. The period now provided for is five years, but it is proposed to buy the land upon which the observatories will be located, or lease it for one hundred years. It is evident that at least twenty-one years would be desirable, because during the seven years of observations already made the pole has returned nearly to its mean position, and three of these cycles should be completed before any definite idea can be had as to its mean path. The cost of the entire work will be about 2000 l. annually. The visual method is to be followed regularly without, however, excluding the possibility of employing later the photographic one, which has already given excellent results. Twelve groups of stars, each comprising eight pairs, will be selected. Six pairs in each group are destined for the latitude determinations proper, while the two remaining pairs, having great zenith distances (about $60^{\circ}$ ), will, it is hoped, throw light on the question of refraction. The observing period for each night is four hours, and will vary from 7 p.m. to 3 a.m., depending on the situation of the group. The instrumental outfit will consist of a zenith telescope and astronomical clock for each station, except that of Japan. Here a chronometer will be substituted for the clock, on account of the frequency of earthquakes.

Although the object of the general conference was scientific discussion, a faithful historian cannot ignore the social and humanitarian side of the function. From our entrance into the beautiful capital of Wurtemberg until the time of our departure we were the recipients of the most cordial hospitality.

Before closing the present paper, attention should be called to a few points of interest noted during the trip to Stuttgart and return. A flying visit was made to the Royal Observatory at Berlin, the Reichsanstalt at Charlottenburg, and the Geodetic Institute at Potsdam. At Paris the offices of the geographic service and the International Bureau of Weights and Measure were examined, and part of one day was devoted to the English Ordnance Survey at Southampton.

An interesting object at the Berlin Observatory is the instrument with which Kiistner discovered the variation of latitude ; not alone because of the splendid result achieved, but on accoun of the conditions under which the work was done. It is mounted on a pier more than twenty feet above ground, on a subsoil of sand, in the middle of a city, with bad atmospheric conditions and about one hundred feet from the public thoroughfares. In spite of these adverse circumstances a new fact was added to science, which had baffled the efforts of larger telescopes under immeasurably better conditions. There is much encouragement in this to investigators with scanty means at their disposal.

At the Aichungs-Kommission a balance was shown which easily determines the weight of a kilogram with an error of $\mathrm{I} / 200$ of a milligram, being $1 / 200,000,000$ part of the quantity sought. They have also a complete series of weights in quartz from $1 / 2$ gram to one kilogram, and thermometers giving the temperature by estimation to $\mathrm{I} / \mathrm{I} 000$ of a degree Centigrade.

At Charlottenburg the most striking feature was the extension and perfection of the organisation. Nine buildings in all, of which the two larger are devoted, one to theory and the other to practice, have cost, together with the running expenses since I $887,3,000,000$ marks. The annual outlay is at present about I $8,000 \%$.

The Geodetic Institute at Potsdam has been much less expensive, and presents many admirable points of arrangement and administration. Among the details may be cited : the clock room, always maintained at a temperature between $20^{\circ}$ and $2 \mathrm{I}^{\circ}$ Centigrade ; the pendulum room, artificially heated on all sides, including the floor; a pillar over fifty feet high, and correspondingly thick, with meridian marks several miles away, to study changes in azimuth and the movement of the earth's crust ; and finally a small photographic instrument, by means of which the occupation of a station only requires eight minutes, and gives a determination of the geographical position in latitude within two seconds of arc. The subsoil, as at Berlin, is nothing but sand.

At Sevres, near Paris, several interesting instruments were seen, among which may be especially mentioned that designed for the comparison of the metre with the wave-length of light following Michelson's method, and the apparatus for the determination of coefficients of expansion according to the method of Fizeau. Some recent experiments have been made on a com. position containing 36 per cent. nickel and 64 per cent. steel. It appears that the expansion from heat is thus reduced to about I/50 of what we should expect from the individual components. This discovery will simplify enormously the solution of problem. where the temperature question has thus far been the great difficulty. It will, for example, be a comparatively easy matter to make pendulum clocks run with a daily correction of about r/ro of a second per day under varying temperature conditions.

\section{MIRAGE. ${ }^{1}$}

WHEN a ray of light passes from point to point or a medium which is everywhere similarly constituted, its path is a straight line; when it passes from one medium to another medium of different density, then the ray of light is refracted or bent at the surface which separates the two media. When the ray passes from one medium to another which is denser, the refraction or bending is always towards the normal to the surface separating the two media at the point of incidence ; when, on the other hand, the ray passes from a medium of a certain density to one of less density, then the bending is always from the normal to the common surface at the point of incidence. The earth is surrounded with a spherical envelope of air, and if that air were always of the same density everywhere its refractive index would be the same, and there would be no terrestrial refraction. But the spherical envelope which surrounds the earth is not all of the same density, and the refractive index of the air varies with the density. There are two causes, in the main, which militate against the uniform density of the atmosphere; one is barometric pressure, and the other is temperature. Taking no account of temperature for the moment, taking merely as the cause barometric pressure, the density of the air diminishes gradually upwards from the surface of the earth, so that the refractive index of the air diminishes upwards. The diminishing of the refractive index is not absolutely proportional to the decrease of density, but it is found by experiment to be sensibly proportional to the excess of the density over unity. The circumstance of normal refraction in the British Isles, as regards temperature, is that there is a gradual diminution of temperature upwards at the rate of about $\mathrm{I} / 300^{\circ} \mathrm{F}$. for every foot of ascent. As the air gets cooler the density increases, so the tendency is to some extent to counteract the effect of barometric pressure, but it does not altogether do so. The result in the normal refraction of the British Isles is that there is a gradual diminution of density upwards.

We may consider the air to be stratified in horizontal layers; as a matter of fact, it is stratified in spherical layers, but it will simplify matters to consider it stratified in horizontal layers, the more so as the sphericity of the earth, though it is a slight cause of terrestrial refraction, is not by any means the chief cause; terrestrial refraction would still exist if the earth had no sphericity, and if its surface were perfectly plane. I show you here a diagram representing the normal state of the atmosphere, and showing the curvilinear path taken by a ray of light when it passes from one point of such an atmosphere to another point horizontally distant from it. The reason a curved path is taken is this : supposing the ray to have a general direction upwards, and supposing it to have been inclined at incidence at a certain angle with the normal, as it is going from a medium-air-to air which is less dense, it bends away from the normal, and therefore there would be a successive bending away from the normal at each layer until finally the ray would arrive at the highest point in the diagram. Then, if it were to pass downwards, it would be passing from a medium of a certain density to one of a greater density, and it would approach the normal at each surface of separation of the media, and therefore its path would be a curved path presenting concavity downwards. A ray of light will actually take.some such path, because by curving upwards it takes the path which it can pass over in the least time. Generally, a ray of light takes the minimum path as regards time, and it is found to curve up into the layers of air which are of less density, because it can traverse them with greater velocity. It is important to notice that a ray of light

1 A lecture, delivered at the Camera Club, by Major P. A. MacMahon, F.R.S.

$$
\text { NO. I 524, VOL. 59] }
$$

